\title{
Overdominant Effect of a CHRNA4 Polymorphism on Cingulo-Opercular Network Activity and Cognitive Control
}

\author{
[- Sepideh Sadaghiani, ${ }^{1,2,3}$ @Bernard Ng, ${ }^{1,4}$ Andre Altmann, ${ }^{1,5}$ Jean-Baptiste Poline, ${ }^{6}$ Tobias Banaschewski, ${ }^{7}$ \\ DArun L.W. Bokde, ${ }^{8}$ Uli Bromberg, ${ }^{9}$ Christian Büchel, ${ }^{9}$ Erin Burke Quinlan, ${ }^{10}$ Patricia Conrod, ${ }^{10}$ Sylvane Desrivières, ${ }^{10}$ \\ -Herta Flor, ${ }^{11,12}$ Vincent Frouin, ${ }^{13}$ Hugh Garavan, ${ }^{14}$ Penny Gowland, ${ }^{15}$ Jürgen Gallinat, ${ }^{16}$ @Andreas Heinz, ${ }^{16}$ \\ Bernd Ittermann, ${ }^{17}$ - $J e a n-L u c$ Martinot, ${ }^{18,19,20,21}$ Marie-Laure Paillère Martinot, ${ }^{19,22}$ - Hervé Lemaitre, ${ }^{18}$ Frauke Nees, ${ }^{7,11}$ \\ ĐDimitri Papadopoulos Orfanos, ${ }^{13}$ Tomáš Paus, ${ }^{23}$ Luise Poustka, ${ }^{24,25}{ }^{\circledR}$ Sabina Millenet, ${ }^{7}$ Juliane H. Fröhner, ${ }^{26}$ \\ Michael N. Smolka, ${ }^{26}$ Henrik Walter, ${ }^{16}$ CRobert Whelan, ${ }^{27}$ Gunter Schumann, ${ }^{10}$ Valerio Napolioni, ${ }^{1}$ \\ and Michael Greicius ${ }^{1}$ \\ ${ }^{1}$ Department of Neurology and Neurological Sciences, Stanford University, Stanford, California 94305, 2Department of Psychology, University of Illinois at \\ Urbana-Champaign, Urbana, Illinois 61801, ${ }^{3}$ Beckman Institute for Advanced Science and Technology, Urbana, Illinois 61801, ${ }^{4}$ Department of Statistics, \\ University of British Columbia, Vancouver BC V6T 1Z4, Canada, ${ }^{5}$ Translational Imaging Group, Centre for Medical Image Computing, Department of \\ Medical Physics and Bioengineering, University College London, London WC1E 6BT, United Kingdom, ${ }^{6}$ Department of Psychology, University of \\ California, Berkeley, California 94720, ${ }^{7}$ Department of Child and Adolescent Psychiatry and Psychotherapy, Central Institute of Mental Health, Medical \\ Faculty Mannheim, Heidelberg University, 68159 Mannheim, Germany, ${ }^{8}$ Discipline of Psychiatry, School of Medicine and Trinity College Institute of \\ Neuroscience, Trinity College, Dublin 2, Ireland, ${ }^{9}$ University Medical Centre Hamburg-Eppendorf, 20246 Hamburg, Germany, ${ }^{10}$ Medical Research Council, \\ Social, Genetic and Developmental Psychiatry Centre, Institute of Psychiatry, Psychology and Neuroscience, King's College London WC2R 2LS, United \\ Kingdom, ${ }^{11}$ Department of Cognitive and Clinical Neuroscience, Central Institute of Mental Health, Medical Faculty Mannheim, Heidelberg University, \\ 68159 Mannheim, Germany, ${ }^{12}$ Department of Psychology, School of Social Sciences, University of Mannheim, 68131 Mannheim, Germany, ${ }^{13}$ NeuroSpin, \\ CEA, Université Paris-Saclay, F-91191 Gif-sur-Yvette, France, ${ }^{14}$ Departments of Psychiatry and Psychology, University of Vermont, Burlington, Vermont \\ 05405, ${ }^{15}$ Sir Peter Mansfield Imaging Centre School of Physics and Astronomy, University of Nottingham, University Park, Nottingham NG7 2RD, United \\ Kingdom, ${ }^{16}$ Department of Psychiatry and Psychotherapy, Campus Charité Mitte, Charité, Universitätsmedizin Berlin, 10117 Berlin, Germany, \\ ${ }^{17}$ Physikalisch-Technische Bundesanstalt, 10587 Berlin, Germany, ${ }^{18}$ Institut National de la Santé et de la Recherche Médicale, INSERM Unit 1000 \\ "Neuroimaging \& Psychiatry", University Paris Sud - Paris Saclay, 91400 Orsay, France, ${ }^{19}$ University Paris Descartes, 75006 Paris, France, ${ }^{20}$ Service \\ Hospitalier Frédéric Joliot, 91400 Orsay, France, ${ }^{21}$ Maison de Solenn, Cochin Hospital, 75014 Paris, France, ${ }^{22}$ AP-HP, Department of Adolescent \\ Psychopathology and Medicine, Maison de Solenn, Cochin Hospital, 75014 Paris, France, ${ }^{23}$ Rotman Research Institute, Baycrest and Departments of \\ Psychology and Psychiatry, University of Toronto, Toronto, Ontario M6A 2E1, Canada, ${ }^{24}$ Department of Child and Adolescent Psychiatry and \\ Psychotherapy, University Medical Centre Göttingen, 37075 Göttingen, Germany, ${ }^{25}$ Clinic for Child and Adolescent Psychiatry, Medical University of \\ Vienna, 1090 Vienna, Austria, ${ }^{26}$ Department of Psychiatry and Neuroimaging Center, Technische Universität Dresden, 01069 Dresden, Germany, and \\ ${ }^{27}$ School of Psychology and Global Brain Health Institute, Trinity College Dublin 2, Ireland
}

The nicotinic system plays an important role in cognitive control and is implicated in several neuropsychiatric conditions. However, the contributions of genetic variability in this system to individuals' cognitive control abilities are poorly understood and the brain processes that mediate such genetic contributions remain largely unidentified. In this first large-scale neuroimaging genetics study of the human nicotinic receptor system (two cohorts, males and females, fMRI total $N=1586$, behavioral total $N=3650$ ), we investigated a common polymorphism of the high-affinity nicotinic receptor $\alpha 4 \beta 2$ (rs1044396 on the CHRNA4 gene) previously implicated in behavioral and nicotine-related studies (albeit with inconsistent major/minor allele impacts). Based on our prior neuroimaging findings, we expected this polymorphism to affect neural activity in the cingulo-opercular (CO) network involved in core cognitive control processes including maintenance of alertness. Consistent across the cohorts, all cortical areas of the CO network showed higher activity in heterozygotes compared with both types of homozygotes during cognitive engagement. This inverted U-shaped relation reflects an overdominant effect; that is, allelic interaction (cumulative evidence $p=1.33 * 10^{-5}$ ). Furthermore, heterozygotes performed more accurately in behavioral tasks that primarily depend on sustained alertness. No effects were observed for haplotypes of the surrounding CHRNA4 region, supporting a true overdominant effect at rs1044396. As a possible mechanism, we observed that this polymorphism is an expression quantitative trait locus modulating CHRNA4 expression levels. This is the first report of overdominance in the nicotinic system. These findings connect CHRNA4 genotype, $\mathrm{CO}$ network activation, and sustained alertness, providing insights into how genetics shapes individuals' cognitive control abilities.

Key words: alertness; cingulo-opercular network; fMRI; genetics; nicotinic acetylcholine receptor; polymorphism 


\section{Significance Statement}

The nicotinic acetylcholine system plays a central role in neuromodulatory regulation of cognitive control processes and is dysregulated in several neuropsychiatric disorders. Despite this functional importance, no large-scale neuroimaging genetics studies have targeted the contributions of genetic variability in this system to human brain activity. Here, we show the impact of a common polymorphism of the high-affinity nicotinic receptor $\alpha 4 \beta 2$ that is consistent across brain activity and behavior in two large human cohorts. We report a hitherto unknown overdominant effect (allelic interaction) at this locus, where the heterozygotes show higher activity in the cingulo-opercular network underlying alertness maintenance and higher behavioral alertness performance than both homozygous groups. This gene- brain- behavior relationship informs about the biological basis of interindividual differences in cognitive control.

\section{Introduction}

Cognitive control abilities are central to all goal-directed behavior but vary widely across individuals (Gruszka et al., 2010; Mennes et al., 2011). Although cognitive control capacities have strong heritable components (Friedman et al., 2008; Chang et al., 2013), it is largely unknown through which brain mechanisms genetic variability translates into their interindividual differences. Neuromodulatory neurotransmitter systems are central to cognitive control given their capacity to modify signal processing broadly across large areas of the brain. In particular, the broad acetylcholinergic innervation of the neocortex originating in the basal forebrain plays a central role in cognitive control, especially tonic control functions (Knott et al., 1999; Kozak et al., 2006). Both tonic control functions and acetylcholinergic modulation are dysregulated in several neuropsychiatric disorders (Lesh et al.,

Received April 12, 2017; revised Aug. 20, 2017; accepted Aug. 22, 2017.

Author contributions: S.S., A.A., V.N., and M.G. designed research;S.S., B.N., A.A., and V.N. performed research; S.S., B.N., A.A., J.-B.P., T.B., A.B., U.B., C.B., E.B.Q., P.C., S.D., H.F., V.F., H.G., P.G., J.G., A.H., B.I., J.-L.M., M.-L.P.M., H.L., F.N., D.P.O., T.P., L.P., S.M., J.F., M.N.S., H.W., R.W., and G.S. contributed unpublished reagents/analytic tools; S.S., B.N., A.A., and V.N. analyzed data; S.S., V.N., and M.G. wrote the paper.

We thank all investigators contributing to the Philadelphia Neurodevelopmental Cohort, especially Theodore D. Satterthwaite, for supporting this work. We thank Stephen M. Malone for supporting us in a multimodal investigation of CHRNA4. This work was supported by funding from The Feldman Family Foundation; the J.W. Bagley Foundation; the Medical Research Council (MRC grant MR/L016311/1). The IMAGEN consortium has received support from the following sources: the European Union-funded FP6 Integrated Project IMAGEN (Reinforcement-related behaviour in normal brain function and psychopathology; LSHM-CT-2007-037286), the Horizon 2020 funded European Research Council Advanced Grant STRATIFY (Brain network based stratification of reinforcement-related disorders; 695313), ERANID (Understanding the Interplay between Cultural, Biological and Subjective Factors in Drug Use Pathways; PR-ST-0416-10004), BRIDGET (JPND: BRain Imaging, cognition Dementia and next generation GEnomics; MR/N027558/1), the FP7 projects IMAGEMEND(602450; IMAging GEnetics for MENtal Disorders) and MATRICS (603016), the Innovative Medicine Initiative Project EU-AIMS (115300-2), the Medical Research Council Grant c-VEDA (Consortium on Vulnerability to Externalizing Disorders and Addictions; MR/N000390/1), the Swedish Research Council FORMAS, the Medical Research Council, the National Institute for Health Research (NIHR) Biomedical Research Centre at South London and Maudsley NHS Foundation Trust and King's College London, the Bundesministerium für Bildung und Forschung (BMBF Grants 01GS08152; 01EV0711; eMED SysAlc01ZX1311A; Forschungsnetz AERIAL), the Deutsche Forschungsgemeinschaft (DFG Grants SM 80/7-1, SM 80/7-2, SFB 940/1). Further support was provided by grants from: Agence Nationale de la Recherche (ANDR project AF12-NEUR000801-WM2NA, and ANR-12-SAMA-0004), the Fondation de France, the Fondation pour la Recherche Médicale, the Mission Interministérielle de Lutte-contre-les-Drogues-et-les-Conduites-Addictives (MILDECA), the AssistancePublique-Hôpitaux-de-Paris and Institut National de la Santé et de la Recherche Médicale (INSERM interface grant), Paris Sud University IDEX 2012; the National Institutes of Health (NIH), Science Foundation Ireland (16/ERCD/3797; Axon, Testosterone and Mental Health during Adolescence; Grant R01 MH085772-01A1), and by NIH Consortium (Grant U54 EB020403), supported by a cross-NIH alliance that funds Big Data to Knowledge Centres of Excellence. A.A. holds an MRC eMedLab Medical Bioinformatics Career Development Fellowship.

T.B. has served as an advisor or consultant to Bristol-Myers Squibb, Desitin Arzneimittel, Eli Lilly, Medice, Novartis, Pfizer, Shire, UCB, and Vifor Pharma; he has received conference attendance support, conference support, or speaking fees from Eli Lilly, Janssen McNeil, Medice, Novartis, Shire, and UCB; and he is involved in clinical trials conducted by Eli Lilly, Novartis, and Shire; the present work is unrelated to these relationships. H.W. received a speaker honorarium from Servier (2014). The remaining authors declare no competing financial interests.

Correspondence should be addressed to Sepideh Sadaghiani, Ph.D., Assistant Professor, Department of Psychology, Cognitive Neuroscience Division, Beckman Institute for Advanced Science and Technology, University of Illinois at Urbana-Champaign, Beckman Institute, 405 N Mathews Avenue, Urbana, IL 61801. E-mail: Sepideh@illinois.edu. DOI:10.1523/JNEUROSCI.0991-17.2017

Copyright $\odot 2017$ the authors $\quad 0270-6474 / 17 / 379658-10 \$ 15.00 / 0$
2011; Sarter and Paolone, 2011; Higley and Picciotto, 2014), reward processing, and addiction to various substances (Hendrickson et al., 2013). However, how genetic polymorphisms in this modulatory system influence brain function is poorly understood.

The most abundant high-affinity nAChR in the mammalian brain is the $\alpha 4 \beta 2$ receptor (Albuquerque et al., 2009). Among the single nucleotide polymorphisms (SNPs) of the underlying genes CHRNA4 and CHRNB2, rs1044396 (NM_000744.6:c.1629C>T) of the $\alpha 4$ subunit (chromosome 20q13.3) has been implicated in behaviorally relevant contexts, albeit with inconsistent impact from major/minor alleles. Although this SNP itself is synonymous (NP_000735.1:p.Ser543=), it is part of a functional CHRNA4 haplotype affecting receptor sensitivity to acetylcholine (Eggert et al., 2015). The SNP is implicated in nicotine consumption and addiction (Feng et al., 2004; Breitling et al., 2009), as well as phasic cognitive control functions. However, this cognitive literature (often comprising relatively small sample sizes) is inconclusive because some studies report a behavioral advantage of the rs1044396-T allele (Espeseth et al., 2010; Greenwood et al., 2012, 2005) and some the rs1044396-C allele (Parasuraman et al., 2005; Reinvang et al., 2009). Furthermore, the brain mechanisms mediating the impact on behavior are largely unknown. The only two neuroimaging investigations of rs1044396 have been performed in relatively small sample sizes $N<50$ and one study lacks heterozygous participants (Winterer et al., 2007; Giessing et al., 2012).

The cortical target regions of acetylcholinergic stimulation may shed light on the underlying pathway from genetic variability to cognitive abilities. Using positron emission tomography, we found that, across the cerebral cortex, $\alpha 4 \beta 2$ receptor density was highest bilaterally in the dorsal anterior cingulate cortex and anterior insula (Picard et al., 2013). Together with the thalamus, the brain region with the highest $\mathrm{nAchR}$ density (Gallezot et al., 2005), these areas constitute the core of the cingulo-opercular (CO) network, also referred to as salience network (see Fig. 2A) (Dosenbach et al., 2006; Seeley et al., 2007). The anatomically selective mapping of $\alpha 4 \beta 2$ receptor density to this network generates a targeted hypothesis regarding the brain structures mediating the cognitive impact of the $\alpha 4$ polymorphism rs 1044396 .

The spatial relation between the CO network and $\alpha 4 \beta 2 \mathrm{nAChR}$ density suggests that functional differences in this receptor may affect the cognitive function of the CO network. Several lines of research suggest that one core cognitive control function of the $\mathrm{CO}$ network is the maintenance of sustained/tonic alertness or vigilance (Sturm et al., 2004; Sadaghiani et al., 2010). Tonic alertness describes the mentally effortful, self-initiated (rather than externally driven), and continuous preparedness to process information and to respond (Parasuraman, 1998; Posner, 2008). A 
Table 1. Demographics and genotype breakdown of included subjects

\begin{tabular}{lllll}
\hline & \multicolumn{3}{l}{ IMAGEN cohort } & \multicolumn{3}{l}{ PNC cohort } \\
\cline { 2 - 3 } & fMRI & Behavioral & fMRI & Behavioral \\
\hline T/T carriers & 354 (189 females) & 403 (209 females) & 66 (37 females) & 608 (333 females) \\
T/C carriers & 671 (340 females) & 751 (383 females) & 111 (55 females) & 1077 (573 females) \\
C/C carriers & 333 (166 females) & 345 (168 females) & 51 (25 females) & 466 (250 females) \\
Total & 1358 (695 females) & 1499 (760 females) & 228 (117 females) & 2151 (1156 females) \\
Age (y) & $14 \pm 0$ & $14 \pm 0$ & $16.9 \pm 1.8$ & $16.7 \pm 1.9$ \\
\hline
\end{tabular}

distinctive characteristic of the CO network is that it becomes active whenever cognitive engagement is required regardless of the specific task (Dosenbach et al., 2006; Yeo et al., 2015), likely due to tonic alertness demands present across cognitive tasks (Sadaghiani and D'Esposito, 2015).

Here, we test the hypothesis that the $\alpha 4 \beta 2 \mathrm{nAChR}$ genotype affects CO network activation during cognitively demanding tasks and explains performance differences in tonic alertness. We focus on the CHRNA4 rs1044396 genotype in light of the abovedescribed prior behavioral literature. We study the impact of this polymorphism on brain activity and behavior in a large dataset in adolescents, with replication in an independent cohort of adolescents and young adults.

\section{Materials and Methods}

Subjects

Adolescents and young adults of Caucasian descent were investigated in two cohorts, IMAGEN and Philadelphia Neurodevelopmental Cohort (PNC), as detailed in Table 1. The IMAGEN cohort contains $>2000$ subjects studied in eight cities across Europe. The cohort and data acquisition have been described in detail previously (Schumann et al., 2010). All subjects were 14 years of age at the time of data collection. We retained all subjects with SNP rs 1044396 imputation accuracy $>0.9$ (see "Genetics" section below). Among these, $n=1499$ subjects had behavioral data in the rapid visual processing task and $n=1358$ subjects had neuroimaging data in the stop signal task (see "fMRI acquisition" section below). Pubertal development stage was determined for use as a covariate using the Puberty Development Scale (Petersen et al., 1988), a self-reported measure of physical development based on the scale introduced by Tanner (1978). On this five-category scale, the vast majority of subjects had a puberty category score of 3 or 4 (median [interquartile range] $=4[1]$ ).

From $>8000$ American subjects studied in Philadelphia for the PNC cohort, all of those who identified as being of Caucasian descent (not including mixed ethnicities) were selected for ethnic homogeneity and comparability with the IMAGEN cohort $(n=4734)$. The cohort and data acquisition were described in detail previously (Satterthwaite et al., 2014, 2016). We retained all subjects with SNP rs 1044396 imputation accuracy $>0.9$. For comparability with the IMAGEN dataset, only subjects of at least 14 years of age were included (range 14-22). Among these, $n=2151$ had behavioral data in the Penn continuous performance test (CPT) experiment and $n=228$ had neuroimaging data in the $n$-back experiment.

\section{Genetics}

IMAGEN subjects were genotyped from blood samples on 610-Quad SNP and 660-Quad SNP arrays from Illumina. The vast majority of PNC subjects were genotyped from blood samples on the 550HH and 610-Quad SNP arrays from Illumina. Because rs1044396 SNP was not included in the Illumina array platforms by the IMAGEN and PNC consortia, we imputed CHRNA4 rs1044396 using the Haplotype Reference Consortium r1.1. as a reference panel (McCarthy, 2016). In the IMAGEN cohort, CHRNA4 rs 1044396 was successfully imputed for $89.3 \%$ of the subjects using the Sanger Imputation Service (https://imputation.sanger.ac.uk/) with EAGLE2 (Loh et al., 2016) and PBWT (Durbin, 2014); minor allele frequency (MAF) was 0.479 , as expected in Caucasians (European 1000 Genomes Consortium Phase $3 \mathrm{MAF}=0.471 ; 1000$ Genomes Project Consortium, 2015). In the PNC cohort, CHRNA4 rs 1044396 was successfully im- puted for $88.4 \%$ of the subjects using the Michigan Imputation Server (https://imputationserver.sph.umich.edu/) (Das et al., 2016) with SHAPEIT2 (Delaneau et al., 2013) and Minimac3 (Das et al., 2016). Note that, although imputation was performed on different servers for the two cohorts, because this process was completed at different instances and sites, both servers used an identical reference set. The MAF was 0.472 . Genotype distribution did not deviate from Hardy-Weinberg equilibrium in the IMAGEN $(p=0.77)$ and PNC $(p=0.99)$ cohorts.

Linkage disequilibrium (LD) analysis was performed using Haploview version 4.2 and defining LD blocks based on the solid spine of LD algorithm (Barrett et al., 2005). Haplotype-based association testing was performed using PLINK by logistic regression model, adjusting for the same covariates used in the analysis of individual datasets. Results from each dataset were fixed-effect meta-analyzed using GWAMA (Mägi and Morris, 2010).

\section{fMRI acquisition}

At IMAGEN sites, structural and functional MRI was performed on $3 \mathrm{~T}$ scanners from a range of manufacturers (at Hamburg, Mannheim, Dresden, and Paris: Siemens Trio with 12-channel head coil, Siemens; at Berlin: Siemens Verio with 8- and 12-channel head coils; at Dublin and Nottingham: Philips Achieva with 8-channel head coil; and at London: GE HDx with 8-channel head coil. A set of imaging sequence parameters compatible with all scanners, particularly those directly affecting image contrast or signal-to-noise, was devised and held constant across sites. Functional imaging parameters consisted of 8 min echoplanar imaging with TR/TE/flip angle $=2200 \mathrm{~ms} / 30 \mathrm{~ms} / 75^{\circ}, 64 \times 64 \times 40$ voxels with $2.4 \mathrm{~mm}$ slice thickness and $1 \mathrm{~mm}$ slice gap and a field of view of $218 \times 218$ $\mathrm{mm}$, yielding isotopic $3.4 \mathrm{~mm}$ voxels. The structural image consists of a T1weighted MPRAGE image of $256 \times 256 \times 160 / 166$ voxels (depending on manufacturer) with a $1.1 \mathrm{~mm}$ isotropic voxel size. Details are provided in Schumann et al. (2010). Functional images in the PNC cohort were recorded on a Siemens TIM trio scanner with 32-channel head coil and consisted of 11.6 min echoplanar imaging with TR/TE/flip angle = $3000 \mathrm{~ms} / 32 \mathrm{~ms} / 90^{\circ}, 64 \times 64 \times 46$ voxels with $3 \mathrm{~mm}$ slice thickness and no slice gap and a field of view of $192 \times 192 \mathrm{~mm}$, yielding isotopic $3 \mathrm{~mm}$ voxels. The structural image consists of a T1-weighted MPRAGE image of $192 \times 256 \times 160$ voxels, with a $0.9 \times 0.9 \times 1 \mathrm{~mm}$ voxel size. Details have been described previously (Satterthwaite et al., 2013, 2014).

\section{Experimental design}

Tasks for fMRI. Both the IMAGEN and PNC datasets included neuroimaging during tasks demanding high cognitive engagement. In the IMAGEN dataset, among four fMRI runs (a functional localizer and three other tasks), we chose to investigate the stop signal task due to its high cognitive control demands. This task requires subjects to press a left or a right button in response to regularly presented visual "go" stimuli (left- or right-pointing arrows, respectively, every 1.6 to $2 \mathrm{~s}$ ), but to withhold response if the go stimulus was followed by a "stop" signal (upward-pointing arrow). The stop signal was presented unpredictably across trials and the time between the foregone go stimulus and the stop signal (stop signal delay) was adjusted continuously during the run so as to keep the individual subject's stop success at $50 \%$. Stop signal delay (range 0-900 ms) was increased or decreased from an initial duration of $150 \mathrm{~ms}$ at the beginning of the experiment in steps of $50 \mathrm{~ms}$ depending on the subject's stop success/failure (Rubia et al., 2005). There were 400 go trials and 87 stop trials.

In the PNC cohort, among the two available fMRI tasks, we chose to investigate the fractal $n$-back task due to its demands on cognitive control (Satterthwaite et al., 2014). In this task, subjects were presented with complex geometric figures (fractals) for $500 \mathrm{~ms}$ at a fixed $2500 \mathrm{~ms}$ interstimulus interval. In different block conditions, subjects pressed a button if they detected a predefined target fractal (0-back condition), if the current fractal was identical to the previous one (1-back condition), or if the current fractal was identical to the fractal 2 trials previously (2-back condition). Visual instructions ( $9 \mathrm{~s}$ ) preceded each block informing the participant of the upcoming condition. Each condition was performed in three blocks of 20 trials $(60 \mathrm{~s})$ each. There were a total of 45 targets and 135 foils with 1:3 ratio in each block. A 24 s passive fixation period was presented at the beginning, middle, and end of the task. 
Tasks for behavioral assessments. CPTs are available as part of larger cognitive test batteries in both cohorts. The Cambridge Neuropsychological Test Automated Battery (CANTAB; http://www.cambridgecognition.com) acquired in the IMAGEN cohort includes the rapid visual processing CPT task. This task requires subjects to detect a predefined target series of 3 digits in a continuous stream of digits (2-9) presented at a rate of $100 / \mathrm{min}$. There were 27 occurrences of the target sequence during the 8 min experimental run. Accuracy in this task is commonly measured using performance accuracy $\left(A^{\prime}\right)$ (Gau and Huang, 2014). $A^{\prime}$ is defined as $0.5+\left[(h-f)+(h-f)^{2}\right] /[4 \times h \times(1-f)]$, where $h$ is the probability of hits and $f$ is the probability of false alarms. $A^{\prime}$ is a signal detection measure of sensitivity to the target regardless of response tendency. It takes into account both hits and false alarms and is directly comparable to the classical index of sensitivity, performance accuracy $\left(d^{\prime}\right)$ (see below; Sahgal, 1987). However, it is based on a nonparametric signal detection model suitable for the rapid visual processing task in which the sensory effects of stimulus triplets may not be well represented by the normal distribution. Difference in $A^{\prime}$ across genotypes was tested using multiple regression.

The Penn Computerized Neurocognitive Battery (Penn CNB) acquired in the PNC cohort includes the Penn Continuous Performance Test (Kurtz et al., 2001). This task presents a stream of seven-segment displays (connected horizontal and vertical lines) at a rate of $60 / \mathrm{min}$. The subjects were required to press a button whenever the display formed a digit (first half of experiment) or a letter (second half of experiment). There were 60 occurrences of targets ( 30 digits and 30 letters) during a total of $6 \mathrm{~min}$. Accuracy was measured as sensitivity to the target regardless of response tendency using the classical sensitivity index $d^{\prime}=Z(h)-$ $Z(f)$, where $Z(p)$ is the inverse of the cumulative distribution function of the Gaussian distribution. Hit rates $(h)$ of 1 were replaced with $(n-$ $0.5) / n$ and false alarm rates $(f)$ of 0 were replaced with $0.5 / n$, where $n$ is the number of targets or nontargets, respectively (Macmillan and Kaplan, 1985). Difference in $d^{\prime}$ across genotypes was tested using multiple regression.

\section{Statistical analysis}

fMRI preprocessing. The fMRI data provided on the IMAGEN database were already slice timing corrected, motion corrected, and spatially normalized to MNI space using SPM8 (http://www.fil.ion.ucl.ac.uk/spm/). For PNC fMRI data, we applied motion correction and spatial normalization to MNI space using ANTs (http://stnava.github.io/ANTs/). Further preprocessing was equivalent across IMAGEN and PNC datasets, which included regressing out six linear head motion parameters, white matter, and CSF confounds (based on segmentation, thresholded at 95\% tissue type probability), five principal components of high variance voxels derived using CompCor (Behzadi et al., 2007), and one-time sampleshifted variants, as well as discrete cosine functions (for high-pass filtering at $1 / 128 \mathrm{~Hz}$ ) of all confound regressors. Our volumes of interest were largescale networks defined using independent component analysis of restingstate functional connectivity in an independent dataset as available in the 90-region FIND lab atlas (Shirer et al., 2012). Large-scale functional networks defined on the basis of their intrinsic connectivity architecture during resting state provide volume delineation unbiased by particular task-related activation. To this end, the use of an independent atlas permits application of the same volume of interest to both cohorts. Note that no resting-state data were available for a subject-specific definition of networks for the majority of IMAGEN subjects. Time courses were extracted from all voxels across the brain areas of each network, averaged to yield one time course per network and normalized to $z$-scores.

In addition to accounting for head motion with the above-described motion parameters, their time-shifted variants and discrete cosine functions, we verified that head motion did not substantially contribute to between-group effects using mean framewise displacement (MFD) as a measure (Power et al., 2012). Relatively few volumes per subject showed displacement $>3$ SDs above the average MFD across all subjects (IMAGEN 16.1 [3.6\%] \pm 30.7 volumes and PNC 10.9 [4.7\%] \pm 15.5 volumes per subject). Further, only few subjects had an MFD $>3$ SDs over the group average MFD (25 [1.8\%] of IMAGEN subjects and 5 [2.2\%] PNC subjects). Therefore, we did not exclude any subjects or
fMRI volumes based on head motion. Direct contrast of MFD across genotypes ensured that head motion did not differ significantly among $\mathrm{T} / \mathrm{T}, \mathrm{T} / \mathrm{C}$, and $\mathrm{C} / \mathrm{C}$ carriers $(~ p>0.4$ for all pairwise $t$ tests in IMAGEN and PNC).

fMRI general linear models. Analyses were performed using in-house MATLAB code. In IMAGEN's stop signal task, successful go trials densely covered the experimental run and thus served as implicit baseline. The time course of all other events; that is, successfully inhibited stop trials, inhibition failures on stop trials, left-right errors on go trials, and errors of omission (not responded in time on go trials) were convolved with the canonical hemodynamic response function to yield regressors of interest. A general linear model was constructed with these regressors for each subject and each network's time course averaged across all the respective voxels (CO, frontoparietal, dorsal attention, and default mode networks) as response. An equivalent GLM analysis was performed for the whole brain using voxelwise time courses as response. The contrast of interest comprised the sum of the respective regression coefficient estimates. Errors of omission were absent in 20\% of participants and very sparse in the other subjects and therefore were excluded from the contrast. At the group level, the resulting contrast value entered multiple regression with genotypes as regressor of interest.

The whole-brain voxelwise statistics in the IMAGEN cohort was derived by restricting the overdominance contrast volume (T/C carriers $>$ other subjects) to the union of all $116 \mathrm{AAL}$ atlas regions as lenient generic gray matter mask and applying an auxiliary uncorrected threshold of $p<$ 0.005 (two-sided $t$ test) followed by cluster-level correction for multiple comparisons. Covariates of no interest were coregressed. The cluster size for this correction was determined using a Monte Carlo simulation with 1000 permutations of randomized genotypes using in-house MATLAB code.

In PNC's $n$-back task, regressors were generated by convolving the canonical hemodynamic response function with the boxcar time course of 0-back, 1-back, and 2-back blocks. In addition, we modeled preblock instructions ( $9 \mathrm{~s}$ ) as an additional regressor of no interest to account for the respective brain processes. A general linear model was constructed with these regressors for each subject and the time course averaged across all the voxels of the network volume of interest as response. The contrast of interest comprised the sum of the regression coefficient estimates of 0-back, 1-back, and 2-back blocks. At the group level, the resulting contrast value was entered into multiple regression as response, with genotypes as regressor of interest.

For data quality assurance, subjects for which the estimated BOLD response in any of the network volumes-of-interest deviated by $>3$ SDs from the mean were excluded from fMRI group statistics ( 33 subjects in IMAGEN, none in PNC).

Group-level regression ( $F M R I$ and behavioral). An initial model compared fMRI signal across rs1044396 genotypes with no a priori assumption on the genetic model of association using two binary regressors to encode genotypes, with the values 00 for T/T, 10 for T/C, and 01 for $\mathrm{C} / \mathrm{C}$. In subsequent models that specifically tested for presence of overdominance, a binary regressor with 1 encoding $\mathrm{T} / \mathrm{C}$ carriers and 0 encoding $\mathrm{T} / \mathrm{T}$ and $\mathrm{C} / \mathrm{C}$ carriers was used, thus testing $\mathrm{T} / \mathrm{C}$ heterozygotes against $\mathrm{T} / \mathrm{T}$ and $\mathrm{C} / \mathrm{C}$ homozygotes. For the IMAGEN cohort, covariates of no interest comprised sex, puberty score, scan site (seven categorical covariates) and population structure (first three principal components). For the PNC cohort, covariates of no interest included sex, age, and population structure (first three principal components).

\section{Results}

CO network activation was investigated using fMRI of tasks that have high cognitive demands known to engage this network (Whelan et al., 2012; Satterthwaite et al., 2013). Behavior was studied using CPTs, the continuous nature of which is specifically designed and widely used to measure tonic alertness or vigilance selectively (Beck et al., 1956; Kurtz et al., 2001). 

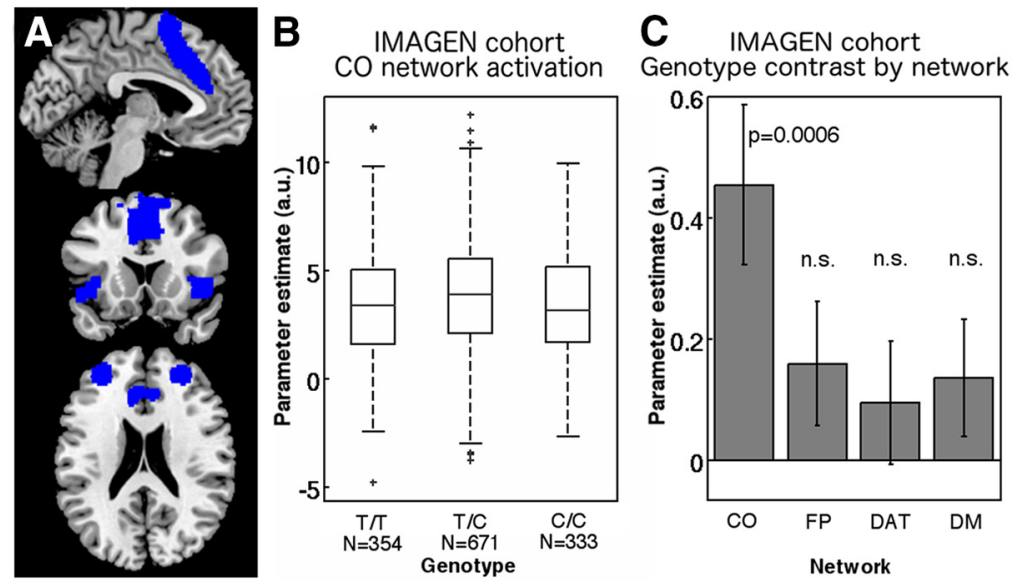

Figure 1. Heterozygotes at the CHRNA4 SNP have increased $C O$ network activation. $A, C O$ network volume of interest in the FINDlab atlas based on intrinsic functional connectivity (Shirer et al., 2012). B. Estimated brain activation averaged across the CO network volume of interest in the IMAGEN cohort during the stop signal task. Higher C 0 network activation is observed in heterozygotes compared with homozygous $\mathrm{T} / \mathrm{T}$ and $\mathrm{C} / \mathrm{C}$ carriers. In boxes, the central mark indicates the median and the bottom and top edges indicate 25th and 75th percentiles, respectively. The whiskers extend to the most extreme data points not considered outliers (within 1.5 interquartile range of the bottom and top of box), and the outliers are marked by " +". C, Genotype contrast $\mathrm{T} / \mathrm{C}>$ homozygotes for activation in the $\mathrm{CO}$ network and three other networks for comparison: FP, Frontoparietal; DAT, dorsal attention; DM, default mode. A significant overdominant effect was observed for the CO network only. Error bars indicate SE.

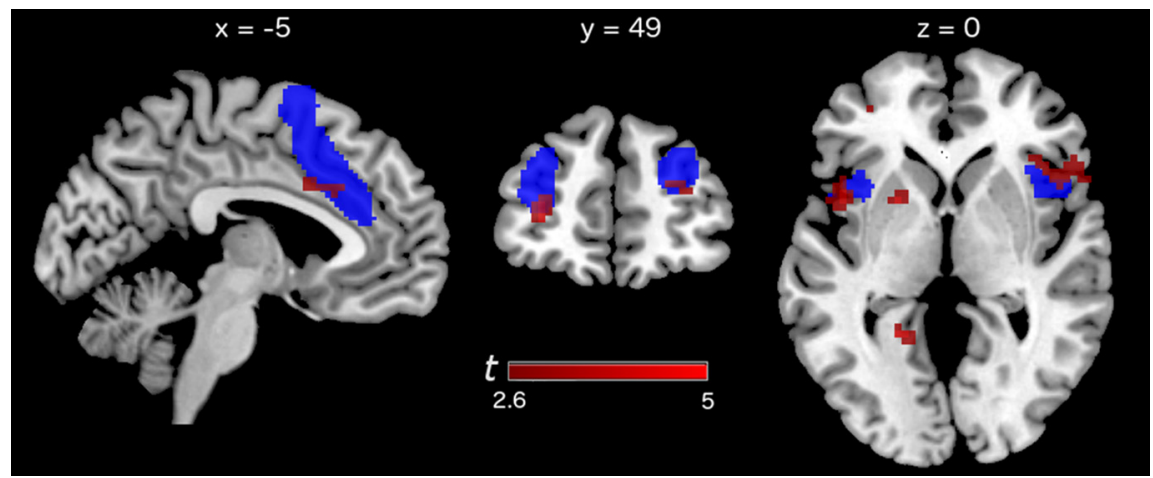

Figure 2. Whole-brain map showing that activation differences across genotypes overlap with the CO network. Shown is the contrast T/C larger than homozygous T/T and C/C carriers in the IMAGEN cohort during the stop signal task ( $p<0.005$ auxiliary uncorrected threshold, corrected at cluster level). Blue shows the $\mathrm{C} 0$ volume of interest as in Figure 1; red shows areas of higher activation in heterozygotes displayed on a canonical single subject structural image demonstrating the overlap in dorsal anterior cingulate, anterior prefrontal, and anterior insula loci.

\section{CHRNA4 polymorphism and CO network activation}

We hypothesized that activity in the CO network during cognitive engagement is affected by rs1044396 genotype. The CO network volume of interest was taken from a functional atlas derived from resting-state functional connectivity analysis of an independent sample (Fig. 1A) (Shirer et al., 2012). In the IMAGEN fMRI dataset ( $n=1358$; Table 1$)$, we investigated network activity during a stop signal task that requires a high level of cognitive control. Subjects had to press a button in response to regularly presented go stimuli but withhold response if the go stimulus was followed by a stop signal. Note that, although this task requires several other cognitive control functions such as top-down inhibition and spatial attention, it is known to heavily involve tonic alertness and the CO network (Satterthwaite et al., 2013). For each subject, the CO network fMRI signal time course was entered in a general linear model comprising regressors for all estimable task events. Estimated brain activity across these events confirmed strong engagement of the CO network volume of interest across all subjects regardless of genotype (one-sample $t$ test $\left.t_{(1357)}=54.57, p<10^{-10}\right)$. With $\mathrm{T} / \mathrm{T}$ (homozygous carriers of the major allele) as the baseline, we examined the effects of the presence of minor allele $\mathrm{C}$; that is, $\mathrm{T} / \mathrm{C}$ and $\mathrm{C} / \mathrm{C}$ genotypes, on $\mathrm{CO}$ network activity using multiple regression with no a priori assumption on the genetic model of association. Taskrelated activity in this network was significantly higher in T/C carriers compared with $\mathrm{T} / \mathrm{T}$ carriers $\left(t_{(1343)}=2.83, p=0.005\right.$; Fig. 1), whereas activity for $\mathrm{C} / \mathrm{C}$ carriers $\mathrm{did}$ not differ from $\mathrm{T} / \mathrm{T}$ carriers $\left(t_{(1343)}=\right.$ $-0.003, p=0.998)$. This result is suggestive of an overdominant effect in which the phenotype of heterozygotes lies outside the phenotypical range of both homozygous groups due to allelic interaction at a single locus (Hochholdinger and Hoecker, 2007). After this observation, we used multiple regression to test specifically for overdominance; that is, T/C carriers $>$ all other subjects. This analysis confirmed higher CO network activity in heterozygotes compared with homozygotes $\left(t_{(1344)}=3.44\right.$, $p=0.0006,0.9 \%$ variance explained).

To test the neuroanatomical specificity of the rs1044396 impact on the CO network, we investigated three other high-level networks as controls. These comprised the default mode network as well as two networks underlying other cognitive control functions, namely the dorsal attention network supporting selective attention and the lateral frontoparietal network supporting phasic adaptive control. Using identical first- and second-level GLM analyses, neither T/C nor $\mathrm{C} / \mathrm{C}$ carriers showed significant differences in network activation compared with T/T carriers in these three control networks (all $t_{(1343)}<$ 1.2), nor was an effect observed when comparing $\mathrm{T} / \mathrm{C}$ against both homozygous groups (all $t_{(1344)}<1.6$; Fig. $1 C$ ).

To further investigate this neuroanatomical specificity, we complemented our volume of interest-based approach with whole-brain voxelwise regression. Contrasting T/C carriers with homozygotes, we found significantly higher activity in T/C carriers across several cortical areas of the CO network (cluster-level corrected based on Monte Carlo permutation test after an auxiliary uncorrected threshold $p<0.005)$. These nodes comprised right and left anterior insulae, right and left anterior prefrontal cortices, and left dorsal anterior cingulate cortex (Fig. 2, Table 2). The clusters showed anatomical overlap and correspondence with all five cortical areas of the $\mathrm{CO}$ network as defined by the FIND atlas (Shirer et al., 2012). We found additional significant clusters largely located in sensory and motor processing regions (Table 2) that may represent task-specific processing top-down modulated by higher cognitive control engagement of the $\mathrm{CO}$ network in heterozygotes.

We tested whether an overdominant effect could be confirmed in the independent PNC fMRI dataset $(n=228)$. This cohort completed an $n$-back task that requires subjects to moni- 
Table 2. Contrasting task-evoked activity between $\mathrm{T} / \mathrm{C}$ carriers and homozygotes

\begin{tabular}{|c|c|c|c|c|c|}
\hline & $\begin{array}{l}\text { MNI } x, y, z \\
\text { coordinates }\end{array}$ & $\begin{array}{l}\text { Peak } \\
t_{(1344)} \\
\end{array}$ & Peak $p$ & $\begin{array}{l}\text { Cluster size } \\
\text { (voxels) }\end{array}$ & $\begin{array}{l}\text { Corrected } \\
\text { cluster } p^{*}\end{array}$ \\
\hline \multicolumn{6}{|l|}{ CO network } \\
\hline \multicolumn{6}{|l|}{ Anterior insula } \\
\hline Right & $3620-5$ & 4.22 & $<5 * 10^{-5}$ & 95 & 0.0004 \\
\hline Left & $-4511-2$ & 4.16 & $<5^{*} 10^{-5}$ & 54 & 0.002 \\
\hline Left & $-3317-8$ & 4.52 & $<5 * 10^{-5}$ & 14 & 0.040 \\
\hline \multicolumn{6}{|l|}{ Anterior prefrontal } \\
\hline Right & 304719 & 3.52 & $<5 * 10^{-4}$ & 14 & 0.040 \\
\hline Left & -30507 & 4.50 & $<5 * 10^{-5}$ & 22 & 0.017 \\
\hline $\begin{array}{l}\text { Dorsal anterior cingulate, } \\
\text { left }\end{array}$ & -62331 & 3.50 & $<5 * 10^{-4}$ & 13 & 0.046 \\
\hline \multicolumn{6}{|l|}{ Non-CO regions } \\
\hline \multicolumn{6}{|l|}{ Precentral gyrus } \\
\hline Left & $-51-1040$ & 4.0 & $<5 * 10^{-5}$ & 38 & 0.005 \\
\hline Right & $33-2549$ & 4.43 & $<5^{*} 10^{-5}$ & 19 & 0.023 \\
\hline Right, inferior & $57-124$ & 3.81 & $<5^{*} 10^{-4}$ & 17 & 0.028 \\
\hline Cuneus, right & $18-7831$ & 3.68 & $<5 * 10^{-4}$ & 30 & 0.010 \\
\hline Lingual gyrus, left & $-18-494$ & 4.16 & $<5^{*} 10^{-5}$ & 28 & 0.010 \\
\hline Putamen, left & -2184 & 3.83 & $<5^{*} 10^{-4}$ & 20 & 0.021 \\
\hline $\begin{array}{l}\text { Superior temporal gyrus, } \\
\text { left }\end{array}$ & $-66-3717$ & 3.83 & $<5 * 10^{-4}$ & 18 & 0.025 \\
\hline
\end{tabular}

*Permutation-based after an auxiliary uncorrected threshold $p<0.005$.

tor a continuous stream of abstract geometric images for specific stimulus repeats. In different block conditions, subjects pressed a button if they detected a predefined target image (0-back condition), if the current image was identical to the previous one (1back condition), or if the current image was identical to the image 2 trials previously (2-back condition). Again, we investigated brain activity evoked by all estimable events (0-back, 1-back, and 2-back trials). Strong engagement of the CO network was confirmed across all subjects regardless of genotype (one-sample $t$ test $t_{(227)}=12.50, p<10^{-10}$ ). Activation in the CO network was then compared across subjects with $\mathrm{rs} 1044396 \mathrm{~T} / \mathrm{T}, \mathrm{T} / \mathrm{C}$, and C/C genotypes (Fig. 3A). Using multiple regression, we tested for overdominance; that is, T/C carriers $>$ all other subjects. This analysis confirmed higher $\mathrm{CO}$ network activation in heterozygotes compared with homozygotes $\left(t_{(221)}=2.77, p=0.006,3.4 \%\right.$ variance explained).

Note that, beyond increased demands on tonic alertness, the $n$-back task requires considerable working memory engagement. This task is thus commonly used to extract working memory processes associated with regions of the frontoparietal network, especially the dorsolateral prefrontal cortex (Owen et al., 2005; D'Esposito and Postle, 2015). Indeed, whereas the frontoparietal network was activated by this task (one-sample $t$ test regardless of genotype $t_{(227)}=4.31, p<10^{-4}$ ), no significant activation difference was found across genotypes in this network or the other two networks, dorsal attention and default mode networks, that we investigated as controls (all $t_{(221)}<0.8$ for T/C against homozygotes; Fig. $3 B$ ). This result again speaks to the anatomical specificity of the impact of rs1044396 on CO network activation.

\section{CHRNA4 rs1044396 and tonic alertness}

After observing that the rs 1044396 polymorphism is associated with the strength of activation in brain areas maintaining tonic alertness, we next investigated whether this impact translates into interindividual differences in behavioral measures of tonic alertness. Tonic alertness, the intrinsically maintained preparedness to process information and to respond, is a necessary prerequisite for more specialized cognitive functions such as selective attention and perceptual processes to build on. In contrast to selective attention and phasic stimulus-driven alertness, tonic alertness is continuous rather than transient (Posner and Boies, 1971) and has a general overarching nature, rather than operating with respect to specific information and sensory features (Robertson and Garavan, 2004).

Note that the tasks for which fMRI data were available coengaged multiple higher-order cognitive processes, rendering the selective investigation of alertness difficult. Therefore, to study behavior, we turned instead to behavioral CPTs that target tonic alertness selectively. The IMAGEN study contains a visual CPT called rapid visual processing, during which subjects $(n=1499)$ continuously attend a visual stream of digits and press a button whenever a predefined target sequence of three digits is detected. $A^{\prime}$ was compared across rs1044396 genotypes (Fig. 4A). Paralleling the neuroimaging findings, we tested for presence of overdominance (i.e., T/C carriers $>$ all other subjects) and found that heterozygotes showed the highest performance accuracy $\left(t_{(1485)}=2.28, p=0.023,0.4 \%\right.$ variance explained $)$. For completeness, we also investigated behavior comprehensively during the fMRI SST task (individual stop signal delay, stop signal reaction time, reaction time on go trials, failures to stop, and left-right errors). We found no significant impact of genotype, presumably because of dependence of performance in this task on multiple overlapping cognitive control faculties, consistent with a lack of behavioral effects during the two previous neuroimaging studies of rs1044396 (Winterer et al., 2007; Giessing et al., 2012).

We then attempted to replicate the presence of overdominance at rs 1044396 on behavior in the independent PNC cohort. PNC uses a visual CPT during which subjects $(n=2151)$ continuously attend a visual stream of figures made of seven lines and press a button whenever the lines form a digit or a letter. $d^{\prime}$ was compared across subjects with rs1044396 T/T, T/C, and C/C genotypes (Fig. 4B). This analysis confirmed higher performance accuracy in heterozygotes compared with T/T and C/C carriers $\left(t_{(2144)}=3.18, p=0.0015,0.5 \%\right.$ variance explained $)$.

\section{Meta-analysis of overdominance}

Finally, to investigate the cumulative evidence gained from IMAGEN and PNC cohorts for overdominance at rs1044396 $(\mathrm{T} / \mathrm{C}>[\mathrm{T} / \mathrm{T} \mathrm{C} / \mathrm{C}])$ in $\mathrm{AMRI}$ and behavioral data, we performed a meta-analysis over the respective effect sizes. We found that $z=$ $4.36, p=1.33 * 10^{-5}$ (total $\left.n=1586\right)$ for the fMRI measures of CO activation and $z=2.54, p=0.011$ (total $n=3650$ ) for behavioral measures of alertness. The behavioral meta-analysis underperformed compared with the AMRI meta-analysis, presumably due to the heterogeneity of the behavioral measure across the two cohorts (behavioral: $q=8.88, p=0.003$; fMRI: $q=$ $0.5, p=0.48)$.

\section{CHRNA4 overdominance and haplotypes}

To further elucidate whether the observed overdominant effect was due to allelic interaction at the SNP of interest or if it was the result of heterozygosity at multiple neighboring locations (pseudooverdominance, see Discussion section), we performed haplotype association tests for the LD block surrounding rs1044396, which includes 28 SNPs. Eleven haplotypes with frequency $>1 \%$ were considered for the analysis. Haplotype frequencies are comparable between IMAGEN and PNC, with the H1 haplotype, which includes the rs 1044396-T allele, being the most frequent (38\%) in both the IMAGEN and PNC cohorts. We found no significant association of CO network activation levels or behavioral measures of alertness for haplotypes of the surrounding CHRNA4 region in either cohort (the omnibus tests were not significant 
A

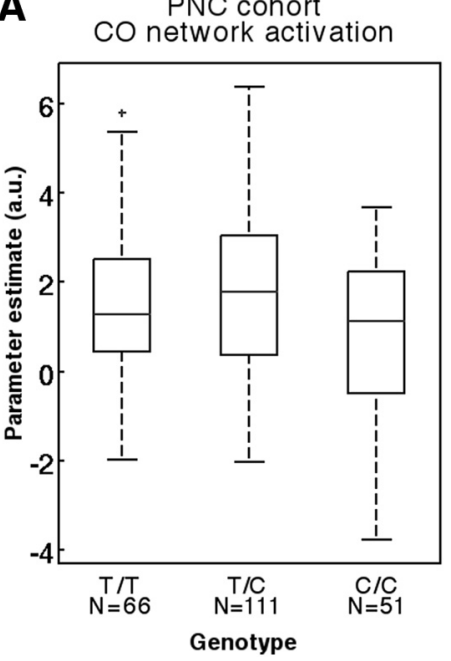

B

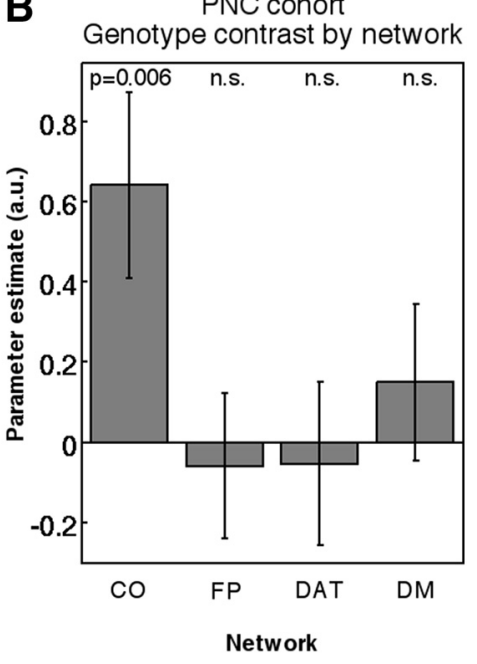

Figure 3. Increased CO network activation in heterozygotes is replicated in the PNC cohort. $\boldsymbol{A}$, Estimated brain activation averaged across the $\mathrm{C} O$ network volume of interest in the PNC cohort during the fractal $n$-back task is shown separately for each genotype. Higher $C 0$ network activation is observed in heterozygotes compared with homozygous $\mathrm{T} / \mathrm{T}$ and $\mathrm{C} / \mathrm{C}$ carriers. Box plots are arranged as explained in Figure 1. $\boldsymbol{B}$, Genotype contrast $\mathrm{T} / \mathrm{C}>$ homozygotes is shown for activation in the $C 0$ network and three other networks for comparison (abbreviations as in Fig. 1). A significant overdominant effect was observed for the CO network only. Error bars indicate SE.
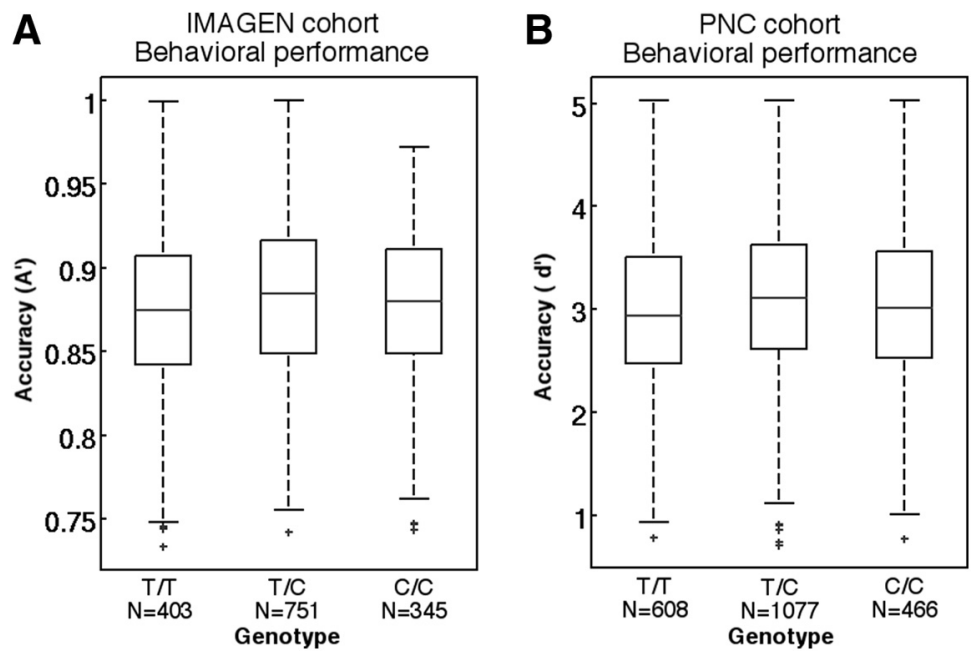

Figure 4. Impact of genotype on tonic alertness capacity shows an overdominant effect. Performance accuracy in CPTs as measured by perceptual sensitivity is shown for the IMAGEN $(\boldsymbol{A})$ and PNC $(\boldsymbol{B})$ cohorts for the three rs 1044396 genotypes. In both datasets, heterozygotes performed better than homozygote carriers of the major (" $\mathrm{T}$ ") or minor (" $\mathrm{C}$ ") allele. Box plots are arranged as explained in Figure 1.

and no individual haplotype showed a significant association). This result speaks against pseudo-overdominance in favor of a true overdominant effect at rs1044396.

\section{CHRNA4 rs1044396 and gene expression levels}

The potential biological mechanisms underlying the observed impact of the synonymous SNP rs 1044396 remains unclear. Although the SNP has no effect on the amino acid level, the change from $\mathrm{T}$ to $\mathrm{C}$ disrupts a potential methylation site (CpG). Indeed, the entire exon 5 of CHRNA4 overlaps with a CpG island (UCSC genome browser; Kent et al., 2002). Therefore, we investigated the dependence of CHRNA4 expression in neural tissue on this polymorphism using publicly available data from the GenotypeTissue Expression (GTEx) project (GTEx Consortium, 2015). Based on the focus of our neuroimaging investigations on large-

scale cortical networks, we investigated the two available cortical regions Brodmann area (BA) 9 (samples = 92; in the vicinity to BA46 that encompasses the anterior prefrontal region of CO network; cf. Fig. $1 A$ ) and BA 24 (samples $=72$; directly overlapping with the anterior cingulate cortex region of the CO network). In addition, we analyzed the tibial nerve because much higher tissue samples were available for it compared with brain tissues (samples $=256$ ). In all investigated neural tissue, we found a linear dosage effect such that homozygous major allele carriers $(\mathrm{T} / \mathrm{T})$ had the highest expression levels and heterozygotes showed intermediate gene expression (BA $9 t=4.3, p=$ $6^{\star} 10^{-5}$, BA $24 t=2.6, p=0.011$; tibial nerve $t=5.4, p=2{ }^{*} 10^{-7}$ ). This analysis shows that rs 1044396 is an expression quantitative trait locus (eQTL) modulating the expression levels of CHRNA4.

\section{Discussion}

Although the nicotinic system plays an important role in cognitive control processes, the contribution of genetic variability in this system to (nicotine consumptionunrelated) cognition has received scant attention (Greenwood et al., 2012). Furthermore, it is not well understood whether any specific brain structures are affected by the genetic makeup of the nicotinic system. Here, we investigated the relation between brain activity and behavior with a common SNP of the most prevalent, highaffinity nicotinic receptor in the brain. Specifically, based on our prior findings of nicotinic receptor distribution (Picard et al., 2013), we expected the rs 1044396 genotype to affect neural activity in the $\mathrm{CO}$ network. In addition, based on the previously established link between the $\mathrm{CO}$ network and sustained alertness (Sadaghiani and D'Esposito, 2015), we expected an impact of this polymorphism on the ability to engage this cognitive control function. The CO network is known to show pervasive activation across numerous distinct cognitive tasks. This general activation profile allowed us to study the $\mathrm{CO}$ network in previously acquired fMRI experiments across two large cohorts. We found that, during cognitive engagement, the CO network, but not other controlrelated networks, showed higher activity in heterozygotes (T/C carriers) compared with homozygous carriers of the major (T/T) or minor allele $(\mathrm{C} / \mathrm{C})$. Furthermore, we observed that heterozygotes performed at significantly higher accuracy in behavioral tasks that primarily depend on the ability to maintain alertness. Findings were consistent across both cohorts totaling $N=1586$ subjects for neuroimaging and $N=3650$ for behavior. These results therefore expand considerably upon encouraging, but relatively underpowered $(N<50)$, neuroimaging studies of this SNP (Winterer et al., 2007; Giessing et al., 2013). One of these 
studies found the highest task-related activity in T/T homozygotes in supplementary motor/anterior cingulate cortex and left postcentral gyrus (Winterer et al., 2007). Conversely, the other study, which did not include heterozygous subjects, found higher activity for $\mathrm{C} / \mathrm{C}$ compared with $\mathrm{T} / \mathrm{T}$ carriers in right middle temporal, but lower activity in right superior temporal gyrus (Giessing et al., 2012). Our results constitute the first report of overdominance in a CHRNA4 association study of brain activity and cognitive performance. This overdominant effect may be one contributor to discrepancy in impact from $\mathrm{T}$ versus $\mathrm{C}$ alleles in previous behavioral and fMRI studies with smaller sample sizes.

\section{Possible mechanisms underlying overdominance}

What could be driving the observed overdominant effect? Overdominance is often missed because the most prevalent genetic models used in genome-wide association studies rely on the a priori assumption that alleles contribute to complex traits in a linear additive fashion. However, overdominance is expected to be very prevalent (Comings and MacMurray, 2000). One common source of overdominance is thought to be the interaction among multimeric protein products (Comings and MacMurray, 2000). The $\alpha 4 \beta 2$ nicotinic receptor is a pentamer and commonly contains two $\alpha 4$ subunits, readily suggesting functional interactions between these subunits. However, rs 1044396 leads to a synonymous amino acid substitution and it seems unlikely that such modification would affect $\alpha 4$ multimerization. A more plausible explanation could relate to a pseudo-overdominant effect (Draghi and Whitlock, 2015) due to the presence of multiple cis-acting CHRNA4 SNPs in the LD block, including rs 1044396, which may favor the expression of a particular haplotype overrepresented in rs 1044396 heterozygotes. However, according to our haplotype analysis, we can exclude the existence of cis-interacting SNPs at the rs1044396-LD block. At the same time, we should not ignore the possibility of a hidden interaction between rs1044396 and another genetic/environmental factor (e.g., a SNP $\times$ SNP interaction or an SNP $\times$ environment interaction). The possibility of an SNP $\times$ environment interaction is supported by the fact that rs 1044396 is followed by a " $G$ " nucleotide, thus creating a potential methylation site $(\mathrm{CpG})$ in rs $1044396 \mathrm{C}$-allele carriers that is absent in rs1044396 T-allele carriers.

\section{Overdominance and functional advantage of intermediate expression levels}

A source for overdominance at rs 1044396 could be an advantage of intermediate CHRNA4 expression levels, possibly modulated by the methylation site. One of the best-known examples of overdominance is the nonsynonymous ( $\mathrm{Val} \rightarrow \mathrm{Met}$ ) SNP rs4680 of the COMT gene. COMT encodes the dopamine-metabolizing enzyme catechol-O-methyltransferase, with the Met variant (T-allele) showing a dosage effect on prefrontal dopamine concentrations. Association of cognitive performance with prefrontal dopamine often follows an inverted $\mathrm{U}$ shape. Therefore, intermediate dopamine levels observed in heterozygous carriers result in better performance in specific cognitive tasks compared with homozygous $\mathrm{C} / \mathrm{C}$ and T/T carriers (Cools and D'Esposito, 2011). An analogous effect could underlie our overdominance observations of CHRNA4, such that having one rs1044396 T-allele would result in intermediate expression levels of the corresponding $\alpha 4$ protein. This interpretation is strongly supported by our finding that rs1044396 is an eQTL for CHRNA4, resulting in intermediate gene expression levels in heterozygotes. Because CHRNA4 likely affects receptor sensitivity to acetylcholine (Eggert et al., 2015), intermediate expression levels might be optimal for certain func- tions such as those underlying maintenance of tonic alertness, resulting in heterosis (superior phenotype of heterozygotes).

The optimal expression level, however, might be dependent on the cognitive function under investigation. In the context of COMT, the ideal prefrontal dopamine level (i.e., the peak of the inverted U-shape function) is task dependent, resulting in discrepancies across COMT association studies (Cools and D'Esposito, 2011). An inverted U function could drive a similar task dependence for rs 1044396 effects and explain the contradictory reports in behavioral association studies (Störmer et al., 2012). Although the high density of $\alpha 4 \beta 2$ receptors in the CO network suggests an especially prominent role of CHRNA4 polymorphisms in sustained alertness, other cognitive control functions are likely affected as well. The association of rs1044396 genotype with performance might differ for tasks that primarily rely on sustained alertness (such as the CPT tasks studied here) compared with those targeting phasic and selective control functions such as spatial attention or cued orienting investigated in previous studies (Greenwood et al., 2005, 2005; Espeseth et al., 2010). Such task dependence may also explain the different findings in the two previous brain imaging studies of rs 1044396 that focused on selective attention tasks (Winterer et al., 2007; Giessing et al., 2012).

\section{Limitations}

One limitation to making use of previously acquired datasets is that we were not able to administer an ideal task specific to tonic alertness during fMRI. Rather, we had to interrogate tonic alertness as a cognitive control function that was common to the cognitively demanding tasks examined here. The available neuroimaging tasks heavily involved more specific functions such as response inhibition (stop signal task in IMAGEN) and working memory ( $n$-back task in PNC) in addition. This coengagement of cognitive functions limits an unequivocal interpretation of the neuroimaging effects as tonic alertness. However, the fact that two very different tasks resulted in comparable overdominant effects supports the interpretation that rs1044396 affects an omnipresent cognitive control function shared across the respective tasks. The observation of overdominant effects in behavioral CPT procedures that selectively target tonic alertness suggests that this general control function might constitute alertness.

Another potential limitation of our study, and a difference from previous association studies of rs1044396, is the subjects' age. The IMAGEN and PNC cohorts consist of adolescents and young adults, whereas the average age in previous behavioral studies has commonly spanned the mid-30 s and higher (Greenwood et al., 2005; Parasuraman et al., 2005; Reinvang et al., 2009). It is conceivable that the genotype effects observed in our cohorts change across the lifespan beyond the age range that we investigated. This question should be addressed in future studies using neuroimaging and genetics cohorts at other ages. A potential difference in the CHRNA4 genotype effect between teenaged subjects and older subjects would provide an important step forward in understanding genetic contributions to individual brain development during puberty.

Finally, the hypothesis-driven investigation of a single common SNP may present a potential limitation in terms of overall functional impact. Common SNPs generally have small effect sizes and are only a small piece of a large picture in the explanation of complex traits and their neural substrate.

\section{Conclusions}

In this association study of the high-affinity nicotinic receptor $\alpha 4 \beta 2$ in two large cohorts, we establish the importance of the CO 
network in mediating the neuromodulatory effects of acetylcholine on cognition. We further provide a piece of the genetic puzzle underlying interindividual differences in the foundational ability to maintain alertness. These insights into the role of genetic variability in brain activation and cognitive control may help us to understand how genetic changes translate into aberrant behavior in various disorders of cognitive control. This line of work may facilitate individualized medicine in the future by informing how particular neuropharmacological treatments will affect individual patients' brain activity and cognition based on their genotype. The specific study of nicotinic receptors can further lend insights into the basis of individuals' susceptibility to nicotine addiction as it depends on brain activity and cognitive control profile. In summary, the current findings establish a connection between CHRNA4 genotype, CO network activation, and sustained alertness, providing insights into brain-behavior relations and how genetics shape them.

\section{References}

Albuquerque EX, Pereira EF, Alkondon M, Rogers SW (2009) Mammalian nicotinic acetylcholine receptors: from structure to function. Physiol Rev 89:73-120. CrossRef Medline

Barrett JC, Fry B, Maller J, Daly MJ (2005) Haploview: analysis and visualization of LD and haplotype maps. Bioinformatics 21:263-265. CrossRef Medline

Beck LH, Bransome ED Jr, Mirsky AF, Rosvold HE, Sarason I (1956) A continuous performance test of brain damage. J Consult Psychol 20:343350. CrossRef Medline

Behzadi Y, Restom K, Liau J, Liu TT (2007) A component based noise correction method (CompCor) for BOLD and perfusion based fMRI. Neuroimage 37:90-101. CrossRef Medline

Breitling LP, Dahmen N, Mittelstrass K, Rujescu D, Gallinat J, Fehr C, Giegling I, Lamina C, Illig T, Müller H, Raum E, Rothenbacher D, Wichmann HE, Brenner H, Winterer G (2009) Association of nicotinic acetylcholine receptor subunit $\alpha 4$ polymorphisms with nicotine dependence in 5500 Germans. Pharmacogenomics J 9:219-224. CrossRef Medline

Chang Z, Lichtenstein P, Asherson PJ, Larsson H (2013) Developmental twin study of attention problems: high heritabilities throughout development. JAMA Psychiatry 70:311-318. CrossRef Medline

Comings DE, MacMurray JP (2000) Molecular heterosis: a review. Mol Genet Metab 71:19-31. CrossRef Medline

Cools R, D'Esposito M (2011) Inverted-U-shaped dopamine actions on human working memory and cognitive control. Biol Psychiatry 69:e113e125. CrossRef Medline

Das S, et al. (2016) Next-generation genotype imputation service and methods. Nat Genet 48:1284-1287. CrossRef Medline

Delaneau O, Zagury JF, Marchini J (2013) Improved whole-chromosome phasing for disease and population genetic studies. Nat Methods 10:5-6. CrossRef Medline

D'Esposito M, Postle BR (2015) The cognitive neuroscience of working memory. Annu Rev Psychol 66:115-142. CrossRef Medline

Dosenbach NU, Visscher KM, Palmer ED, Miezin FM, Wenger KK, Kang HC, Burgund ED, Grimes AL, Schlaggar BL, Petersen SE (2006) A core system for the implementation of task sets. Neuron 50:799-812. CrossRef Medline

Draghi J, Whitlock MC (2015) Overdominance interacts with linkage to determine the rate of adaptation to a new optimum. J Evol Biol 28:95-104. CrossRef Medline

Durbin R (2014) Efficient haplotype matching and storage using the positional Burrows-Wheeler transform (PBWT). Bioinformatics 30:12661272. CrossRef Medline

Eggert M, Winterer G, Wanischeck M, Hoda JC, Bertrand D, Steinlein O (2015) The nicotinic acetylcholine receptor alpha 4 subunit contains a functionally relevant SNP Haplotype. BMC Genet 16:46. CrossRef Medline

Espeseth T, Sneve MH, Rootwelt H, Laeng B (2010) Nicotinic receptor gene CHRNA4 interacts with processing load in attention. PLoS One 5:e14407. CrossRef Medline

Feng Y, Niu T, Xing H, Xu X, Chen C, Peng S, Wang L, Laird N, Xu X (2004) A common haplotype of the nicotine acetylcholine receptor $\alpha 4$ subunit gene is associated with vulnerability to nicotine addiction in men. Am J Hum Genet 75:112-121. CrossRef Medline

Friedman NP, Miyake A, Young SE, Defries JC, Corley RP, Hewitt JK (2008) Individual differences in executive functions are almost entirely genetic in origin. J Exp Psychol Gen 137:201-225. CrossRef Medline

Gallezot JD, Bottlaender M, Grégoire MC, Roumenov D, Deverre JR, Coulon C, Ottaviani M, Dollé F, Syrota A, Valette H (2005) In vivo imaging of human cerebral nicotinic acetylcholine receptors with 2-18F-fluoro-A85380 and PET. J Nucl Med 46:240-247. Medline

Gau SS, Huang WL (2014) Rapid visual information processing as a cognitive endophenotype of attention deficit hyperactivity disorder. Psychol Med 44:435-446. CrossRef Medline

Giessing C, Neber T, Thiel CM (2012) Genetic variation in nicotinic receptors affects brain networks involved in reorienting attention. Neuroimage 59:831-839. CrossRef Medline

Giessing C, Thiel CM, Alexander-Bloch AF, Patel AX, Bullmore ET (2013) Human brain functional network changes associated with enhanced and impaired attentional task performance. J Neurosci 33:5903-5914. CrossRef Medline

Greenwood PM, Fossella JA, Parasuraman R (2005) Specificity of the effect of a nicotinic receptor polymorphism on individual differences in visuospatial attention. J Cogn Neurosci 17:1611-1620. CrossRef Medline

Greenwood PM, Parasuraman R, Espeseth T (2012) A cognitive phenotype for a polymorphism in the nicotinic receptor gene CHRNA4. Neurosci Biobehav Rev 36:1331-1341. CrossRef Medline

Gruszka A, Matthews G, Szymura B (2010) Handbook of individual differences in cognition: attention, memory, and executive control. New York: Springer Science and Business Media.

Hendrickson LM, Guildford MJ, Tapper AR (2013) Neuronal nicotinic acetylcholine receptors: common molecular substrates of nicotine and alcohol dependence. Front Psychiatry 4:29. CrossRef Medline

Higley MJ, Picciotto MR (2014) Neuromodulation by acetylcholine: examples from schizophrenia and depression. Curr Opin Neurobiol 29:88-95. CrossRef Medline

Hochholdinger F, Hoecker N (2007) Towards the molecular basis of heterosis. Trends Plant Sci 12:427-432. CrossRef Medline

Kent WJ, Sugnet CW, Furey TS, Roskin KM, Pringle TH, Zahler AM, Haussler D (2002) The Human Genome Browser at UCSC. Genome Res 12:996-1006. CrossRef Medline

Knott V, Bosman M, Mahoney C, Ilivitsky V, Quirt K (1999) Transdermal nicotine: single dose effects on mood, EEG, performance, and eventrelated potentials. Pharmacol Biochem Behav 63:253-261. CrossRef Medline

Kozak R, Bruno JP, Sarter M (2006) Augmented prefrontal acetylcholine release during challenged attentional performance. Cereb Cortex 16:917. Medline

Kurtz MM, Ragland JD, Bilker W, Gur RC, Gur RE (2001) Comparison of the continuous performance test with and without working memory demands in healthy controls and patients with schizophrenia. Schizophr Res 48:307-316. CrossRef Medline

Lesh TA, Niendam TA, Minzenberg MJ, Carter CS (2011) Cognitive control deficits in schizophrenia: mechanisms and meaning. Neuropsychopharmacology 36:316-338. CrossRef Medline

Loh PR, Danecek P, Palamara PF, Fuchsberger C, A Reshef Y, K Finucane H, Schoenherr S, Forer L, McCarthy S, Abecasis GR, Durbin R, L Price A (2016) Reference-based phasing using the Haplotype Reference Consortium panel. Nat Genet 48:1443-1448. CrossRef Medline

Macmillan NA, Kaplan HL (1985) Detection theory analysis of group data: estimating sensitivity from average hit and false-alarm rates. Psychol Bull 98:185-199. CrossRef Medline

Mägi R, Morris AP (2010) GWAMA: software for genome-wide association meta-analysis. BMC Bioinformatics 11:288. CrossRef Medline

McCarthy S (2016) A reference panel of 64,976 haplotypes for genotype imputation. Nat Genet 48:1279-1283. CrossRef Medline

Mennes M, Zuo XN, Kelly C, Di Martino A, Zang YF, Biswal B, Castellanos FX, Milham MP (2011) Linking inter-individual differences in neural activation and behavior to intrinsic brain dynamics. Neuroimage 54: 2950-2959. CrossRef Medline

Owen AM, McMillan KM, Laird AR, Bullmore E (2005) N-back working memory paradigm: A meta-analysis of normative functional neuroimaging studies. Hum Brain Mapp 25:46-59. CrossRef Medline

Parasuraman R (1998) The attentive brain. Cambridge, MA: MIT. 
Parasuraman R, Greenwood PM, Kumar R, Fossella J (2005) Beyond heritability neurotransmitter genes differentially modulate visuospatial attention and working memory. Psychol Sci 16:200-207. CrossRef Medline

Petersen AC, Crockett L, Richards M, Boxer A (1988) A self-report measure of pubertal status: reliability, validity, and initial norms. J Youth Adolesc 17:117-133. CrossRef Medline

Picard F, Sadaghiani S, Leroy C, Courvoisier DS, Maroy R, Bottlaender M (2013) High density of nicotinic receptors in the cingulo-insular network. Neuroimage 79:42-51. CrossRef Medline

Posner MI (2008) Measuring alertness. Ann N Y Acad Sci 1129:193-199. CrossRef Medline

Posner MI, Boies SJ (1971) Components of attention. Psychol Rev 78:391408. CrossRef

Power JD, Barnes KA, Snyder AZ, Schlaggar BL, Petersen SE (2012) Spurious but systematic correlations in functional connectivity MRI networks arise from subject motion. Neuroimage 59:2142-2154. CrossRef Medline

Reinvang I, Lundervold AJ, Rootwelt H, Wehling E, Espeseth T (2009) Individual variation in a cholinergic receptor gene modulates attention. Neurosci Lett 453:131-134. CrossRef Medline

Robertson IH, Garavan H (2004) Vigilant attention. In: The cognitive neurosciences, Ed 3, pp 563-578. Cambridge, MA: MIT.

Rubia K, Smith AB, Brammer MJ, Toone B, Taylor E (2005) Abnormal brain activation during inhibition and error detection in medicationnaive adolescents with ADHD. Am J Psychiatry 162:1067-1075. CrossRef Medline

Sadaghiani S, D'Esposito M (2015) Functional characterization of the cingulo-opercular network in the maintenance of tonic alertness. Cereb Cortex 25:2763-2773. CrossRef Medline

Sadaghiani S, Scheeringa R, Lehongre K, Morillon B, Giraud AL, Kleinschmidt A (2010) Intrinsic connectivity networks, alpha oscillations, and tonic alertness: a simultaneous electroencephalography/functional magnetic resonance imaging study. J Neurosci 30:10243-10250. CrossRef Medline

Sahgal A (1987) Some limitations of indices derived from signal detection theory: evaluation of an alternative index for measuring bias in memory tasks. Psychopharmacology (Berl) 91:517-520. CrossRef Medline

Sarter M, Paolone G (2011) Deficits in attentional control: cholinergic mechanisms and circuitry-based treatment approaches. Behav Neurosci 125:825-835. CrossRef Medline

Satterthwaite TD, Elliott MA, Gerraty RT, Ruparel K, Loughead J, Calkins ME, Eickhoff SB, Hakonarson H, Gur RC, Gur RE, Wolf DH (2013) An improved framework for confound regression and filtering for control of motion artifact in the preprocessing of resting-state functional connectivity data. Neuroimage 64:240-256. CrossRef Medline
Satterthwaite TD, Elliott MA, Ruparel K, Loughead J, Prabhakaran K, Calkins ME, Hopson R, Jackson C, Keefe J, Riley M, Mentch FD, Sleiman P, Verma R, Davatzikos C, Hakonarson H, Gur RC, Gur RE (2014) Neuroimaging of the Philadelphia Neurodevelopmental Cohort. Neuroimage 86:544-553. CrossRef Medline

Satterthwaite TD, Connolly JJ, Ruparel K, Calkins ME, Jackson C, Elliott MA, Roalf DR, Ryan Hopsona KP, Behr M, Qiu H, Mentch FD, Chiavacci R, Sleiman PM, Gur RC, Hakonarson H, Gur RE (2016) The Philadelphia Neurodevelopmental Cohort: a publicly available resource for the study of normal and abnormal brain development in youth. Neuroimage 124: 1115-1119. CrossRef Medline

Schumann G, et al. (2010) The IMAGEN study: reinforcement-related behaviour in normal brain function and psychopathology. Mol Psychiatry 15:1128-1139. CrossRef Medline

Seeley WW, Menon V, Schatzberg AF, Keller J, Glover GH, Kenna H, Reiss AL, Greicius MD (2007) Dissociable intrinsic connectivity networks for salience processing and executive control. J Neurosci 27:2349-2356. CrossRef Medline

Shirer WR, Ryali S, Rykhlevskaia E, Menon V, Greicius MD (2012) Decoding subject-driven cognitive states with whole-brain connectivity patterns. Cereb Cortex 22:158-165. CrossRef Medline

Störmer VS, Passow S, Biesenack J, Li SC (2012) Dopaminergic and cholinergic modulations of visual-spatial attention and working memory: insights from molecular genetic research and implications for adult cognitive development. Dev Psychol 48:875-889. CrossRef Medline

Sturm W, Longoni F, Fimm B, Dietrich T, Weis S, Kemna S, Herzog H, Willmes K (2004) Network for auditory intrinsic alertness: a PET study. Neuropsychologia 42:563-568. CrossRef Medline

Tanner JM (1978) Growth at adolescence, Ed 2. Springfield, IL: Blackwell Science.

1000 Genomes Project Consortium (2015) A global reference for human genetic variation. Nature 526:68-74. CrossRef Medline

GTEx Consortium (2015) The Genotype-Tissue Expression (GTEx) pilot analysis: multitissue gene regulation in humans. Science 348:648-660. CrossRef Medline

Whelan R, et al. (2012) Adolescent impulsivity phenotypes characterized by distinct brain networks. Nat Neurosci 15:920-925. CrossRef Medline

Winterer G, Musso F, Konrad A, Vucurevic G, Stoeter P, Sander T, Gallinat J (2007) Association of attentional network function with exon 5 variations of the CHRNA4 gene. Hum Mol Genet 16:2165-2174. CrossRef Medline

Yeo BT, Krienen FM, Eickhoff SB, Yaakub SN, Fox PT, Buckner RL, Asplund CL, Chee MW (2015) Functional specialization and flexibility in human association cortex. Cereb Cortex 25:3654-3672. CrossRef Medline 